\title{
Prensa y transición a la democracia. El caso español ${ }^{1}$
}

\author{
Baldemar Hernández Márquez, profesor de la Universidad Juárez Autónoma de Tabasco \\ Luis Núñez Ladevéze, catedrático de Periodismo y director del Instituto de Estudios de Democracia
}

Resumen:

Esta investigación analiza el papel de los medios escritos en el proceso de transición hacia la democracia en el caso español, durante el periodo 1976-1978. Fueron elegidos como referentes fundamentales dos de los periódicos de mayor influencia durante esta etapa: $A B C$ y El País. Se concluyó que ambos periódicos podían ser definidos como defensores de la democracia, aunque $A B C$ sembró dudas sobre la capacidad de los partidos políticos para organizar y garantizar la estabilidad política, social y económica. Por su parte, $E l$ País manifestó sus dudas sobre la total voluntad del gobierno para certificar un transparente desarrollo del proceso electoral.

Palabras clave:

Transición, democracia, política, prensa escrita, mensajes periodísticos.

Abstract:

This research refers to the roles of the Printing Press into the process of transition of Democracy during the period 1976-1978. There were chosen as a dominant reference two of the major influence Newspaper during that stage: ABC and El País. It was conclude that both Newspapers were defined as a "defenders" of democracy. Nevertheless, $\mathrm{ABC}$ created doubts about the police parties' ability to organize and have guarantee in the social, politics and economic stability. On the other hand, El País used to have uncertainty of the total disposal of government to certify a clear or transparent development into the electoral process:

Key Words:

Transition, Democracy, Politics, Press, News Messages.

Esta nota se basa en la Tesis Doctoral sustentada por el profesor Baldemar Hernández Márquez, en la Facultad de Ciencias de la Información de la Universidad Complutense de Madrid, febrero de 2002. 


\section{Objetivos de la investigación}

El objetivo de la investigación obedece a la preocupación que compartimos muchos hispanoamericanos sobre el proceso de globalización económica, que nos obliga a reflexionar serenamente sobre el futuro de nuestros países, en donde tenemos que aprender a vivir, como pensaba Ortega y Gasset, de acuerdo con el tiempo y las circunstancias. Para tener un papel activo en el proceso mundial de globalización económica es necesario establecer una sociedad madura, lo cual obliga a abrirse a la pluralidad de ideas y reforzar los instrumentos que aseguren la estabilidad democrática.

Pluralidad de ideas y estabilidad democrática dependen en gran parte de la función que desempeñan los medios de comunicación, instrumentos imprescindibles para expresarla.

Nuestra tesis se ha ocupado de analizar el papel que ha tenido la prensa española en la etapa de transición a la democracia durante el periodo comprendido entre 1976-1978. El objetivo era tratar de encontrar un modelo de transición política, que pueda servir en nuestros países como marco referencial del conocimiento de este proceso, asumiendo como premisa general, que dadas las afinidades que guardamos con el pueblo español, nos puede ser útil el conocimiento de la función que desempeñan los medios de comunicación en un proceso de transición.

\section{Hipótesis de trabajo}

En el desarrollo de este trabajo de investigación se ha tratado de aprobar la siguiente hipótesis de trabajo:

Ha habido una etapa de transición a la democracia de la sociedad y se ha tratado de demostrar hasta qué punto las directrices en los editoriales de los periódicos de referencia han sido tenidas en cuenta durante el proceso. A estos efectos hemos establecido una tipología de los textos:

- Editoriales de anticipación, en los que se habla de lo que debe ocurrir;

- Editoriales descriptivos, que no opinan, que solo comentan;

- Editoriales que critican ciertas frases y promueven otras cosas;

- Editoriales que solo aprueban lo hecho.

La necesidad de delimitar el material objeto de la investigación llevó a centrarnos en los dos periódicos de mayor referencia, uno de ellos caracterizado por su destacada influencia en los medios conservadores, de 
línea eminentemente monárquica, como el periódico $A B C^{2}$, con casi un siglo de ejercicio periodístico el periodismo; y otro periódico, El País, que nació como una segunda empresa periodística, tomando como antecedentes los espacios de progresismo que permitió la Ley de Prensa de 1966. El periódico $A B C^{3}$ ha guardado con suma discreción una tendencia independiente dentro del gusto y referencias del público lector, compuesto tradicionalmente por familias de inclinación conservadora, entre las que ha ejercido gran influencia, sobre todo en la población mayor de 45 años y con instrucción de secundaria en su gran mayoría. Esto no quiere decir que estemos afirmando que no haya una parte de la población con instrucción universitaria o destacados intelectuales que sean sus asiduos lectores.

En cambio, el público lector del diario El País ${ }^{4}$, desde su aparición por primera vez el 4 de mayo de 1976, es preferentemente joven y con instrucción universitaria. Desde un principio representó a una porción sobresaliente de estos estratos poblacionales. A los pocos meses de su aparición, logró colocarse dentro de un segmento numeroso del mercado en el gusto y preferencias del público lector, que se reflejó con el constante aumento de su circulación. En apenas seis meses, tiró más de 128 mil ejemplares diariamente.

\section{Metodología de la investigación}

Una de las dificultades mayores que se presentaron en el proceso de la investigación fue la de decidir el planteamiento metodológico para llevar a cabo un trabajo sistemático y ordenado que pudiera servir de base para describir, a través del tipo de mensaje de la prensa diaria, un modelo de influencia de la prensa en el proceso de transición política. Inicialmente se hizo una revisión bibliográfica intensa que pudiera servir de referencia a las pautas metodológicas que deberíamos seguir.

Una vez definidos los objetivos y la hipótesis de trabajo de la investigación, se presentó el problema de cómo analizar el material de los mensajes que se iban a estudiar de los periódicos previamente seleccionados, durante los tres años de las etapas de transición a la democracia.

2 El $A B C$ nace con periodicidad inicialmente semanal, el $1^{\circ}$ de enero de 1903.

3 Cabe señalar que no se va a realizar una investigación exhaustiva sobre el periódico $A B C$, para ello se remite al lector interesado en este tema a que consulte la Tesis Doctoral de Francisco Iglesia: "Historia de una empresa periodística (1891-1978)", Editorial Prensa Española, S. A., Madrid, 1980.

4 En su primera publicación, El País apunta en su editorial firmado por Juan Luis Cebrián: “Desde las fechas lejanas en que un grupo de periodistas intelectuales españoles se les ocurriera la idea de fundar El País, éste ha soñado siempre a sí mismo como un periódico independiente capaz de rechazar las presiones que el poder político y el dinero ejercen sobre el mundo de la información”, El País, martes 4 de mayo de 1976. 
En la fase de revisión del material periodístico se optó por clasificar los editoriales y artículos de opinión relacionados con la transición a la democracia en el periodo 1976- 1978. Pero se encontró que en los tres años eran más de dos mil documentos los que se tendrían que examinar uno a uno, por lo que se optó por la realización de un muestreo estadístico (García Lahinguera, 1975: 27-39) en tres etapas. Primero se escogió una muestra del número de periódicos que fuera representativa estadísticamente, partiendo en la selección de un intervalo de confianza del 99,7 \% que representó aproximadamente un margen de error de = 3 desviaciones típicas. Con esto, se pretendía tener una mayor seguridad de elegir efectivamente los artículos con mensajes relacionados con el tema de la transición a la democracia en el periódico de estudio.

En una segunda etapa, con el apoyo de números aleatorios, se escogieron los días y los meses de los diarios que se iban a observar dentro del conglomerado, en el lapso 1976-1978. Posteriormente, en una tercera etapa, una vez seleccionado el número de ejemplares, se clasificaron los editoriales y artículos de opinión de acuerdo con el tipo de mensajes, aplicando las categorías previamente determinadas.

\section{Materiales y métodos}

En la primera etapa se obtuvo una muestra de los tres años (1976-1978) de 396 diarios. Posteriormente, en la segunda etapa, con el apoyo de una tabla de números aleatorios se escogieron únicamente 380 artículos; sin embargo, al examinar el material elegido en la tercera etapa, se descubrió que más de un 20 \% del tipo de mensajes de los editoriales y artículos de opinión no estaban relacionados con el tema de transición a la democracia, por lo que se procedió a depurar el material siguiendo cuatro criterios fundamentales: 1. editoriales y artículos de opinión de anticipación, en los que solo se habla de lo que debe ocurrir; 2. editoriales y artículos de opinión descriptivos, que no opinan, que solo comentan; 3. editoriales y artículos de opinión que critican ciertas fases de la transición y promueven otra cosa; y 4. editoriales y artículos de opinión que aprueban lo hecho. De acuerdo con esta situación, se clasificaron únicamente 305 documentos en base al tipo de mensaje, aplicando las categorías que se mencionan a continuación:

1. editoriales y artículos a favor de la transición a la democracia;

2. editoriales y artículos parcialmente favorables a la transición a la democracia;

3. editoriales y artículos en contra de la transición a la democracia;

4. editoriales y artículos ambivalentes en la transición a la democracia;

5. editoriales y artículos con una línea de tendencia: 
a) de ruptura con el franquismo en la transición a la democracia;

b) sin ruptura con el franquismo en la transición a la democracia; y

c) con reformas en el franquismo en la transición ala democracia.

\section{Métodos usados en el análisis de contenido}

Para el análisis de contenido de los editoriales y artículos de opinión, se utilizó el método propuesto por Violette Morin (1974), consistente en el análisis de aquello que se repite con mayor regularidad en el mensaje, tomando como referencia del análisis de contenido la exhaustividad. Se parte fundamentalmente de la base conceptual de las unidades de información en el contexto del mensaje, que sirvió como material de trabajo para el análisis de contenido del tipo de mensaje, de lo que solo se tomaron en cuenta por su extensión las categorías en las que se concentró mayor número de unidades de información:

a) editoriales y artículos de opinión a favor de la democracia del diario $A B C$ y El País;

b) editoriales y artículos de opinión con una línea de tendencia sin ruptura con el franquismo en la transición a la democracia;

c) editoriales y artículos de opinión con una línea de tendencia de ruptura con el franquismo en la transición a la democracia.

\section{Conclusiones}

En esta Tesis Doctoral se ha tratado de demostrar que las directrices comprendidas en los editoriales y artículos de los periódicos de referencia dominantes desempeñaron un papel muy importante, en la etapa de transición democrática en España, en el periodo 1976-1978.

Una de las cuestiones que más se debatieron en los editoriales y artículos de opinión de ambos diarios, fue el gran número de partidos que participaron en la contienda electoral del 15 de junio de 1977. Los catalogados como mini partidos fueron los más criticados.

Las unidades de información de los mensajes que más destacaron por su elevado grado de politización fueron: "El País que queremos”, "Partidos Políticos” y “Transición Democrática”.

Mientras que los artículos de la tercera página del $A B C$ se dedicaron, en un gran porcentaje, a criticar el papel de los partidos de oposición, por su participación en los comicios, así como al crecido número de los 
llamados mini partidos, en el diario El País los editoriales y artículos de opinión se inclinaron más a criticar la posición del Gobierno no por la intervención de miembros destacados de su gabinete en algunos partidos y en la organización de la jornada electoral.

Generalizando, podría decirse que tanto el diario $A B C$ como El País, se definieron por una clara posición a favor de la democracia. Así lo reflejan los 91 ejemplares que se registraron bajo esta categoría de mensajes, que representó el 30 \% del total de los editoriales y artículos de opinión de la muestra seleccionada de los dos diarios, aproximadamente uno de cada tres mensajes.

En el vasto número de los artículos de la tercera página del $A B C$ predominaron los favorables a una transición a la democracia sin ruptura con el franquismo: 70 de 160 seleccionados, aproximadamente uno de cada dos.

En contraposición, en la generalidad de los editoriales y artículos de opinión de El País hubo una clara tendencia favorable a una transición a la democracia de ruptura con el franquismo, es decir, 66 de los 160 clasificados, o sea, el $44 \%$, que coincidentemente representa casi uno de cada dos.

Por su grado de orientación y compromiso se observó con destacado índice absoluto y ponderado las categorías denominadas: "Monarquía como símbolo" de unidad y la de "Estado de Derecho", que se traduce como sinónimo de armonía y estabilidad social.

Los mensajes de ambivalencia de transición a la democracia sumaron 38 entre los dos diarios (20 del $A B C$ y 18 de El País) o sea el 12 \%, es decir, uno de cada diez. El $A B C$ refleja su ambivalencia en tres aspectos fundamentales:

1. Se dudaba que hubiera un sistema alternativo surgido de la voluntad popular que pudiera sustituir al antiguo régimen franquista que garantizara la estabilidad económica y social, en opinión de algunos de sus articulistas;

2. Se tenía una actitud ambivalente sobre la capacidad de los partidos políticos para organizar y garantizar la estabilidad social, política y económica en el proceso de transición a la democracia.

3. Se presumía que la mayoría de los españoles no contaban con la preparación suficiente para asimilar con madurez y participar en los sufragios, con un voto razonado y encaminado a fortalecer el establecimiento de una democracia participativa; El País contrastaba su ambivalencia en dos puntos de vista, a su juicio, importantes:

a) Se dudaba de la buena disposición del Gobierno para garantizar un proceso transparente y equitativo en la contienda electoral; 
b) Se miraba con mucha desconfianza a la Ley Electoral, considerando que en su momento favorecía más a los partidarios de la Política del Gobierno y que no se respetaría el sufragio de la voluntad popular.

A pesar de las diferencias entre los editoriales y artículos de los diarios El País y ABC, ambos coincidieron en señalar que una de las medidas más necesarias para la transición a la democracia era la legitimación de los partidos políticos. Como respuesta a esta situación, en abril de 1977 el Gobierno permite la legalización del Partido Comunista Español (PCE), con Santiago Carrillo a la cabeza.

Contra los pronósticos de las encuestas ampliamente comentados en los editoriales y artículos de opinión del ABC y El País, de que casi la mitad de los españoles en edad de votar no se presentarían a los comicios, la realidad demostró que la gran mayoría de la población participó en la jornada electoral del 15 de junio de 1977.

Los editoriales y artículos de los diarios $A B C$ y El País esperaban que más del 50 \% de la población no votara en la jornada electoral del 15 de junio de 1977. Sin embargo, casi 18 millones de españoles ejercieron su derecho al voto y la victoria fue para Adolfo Suárez y la Unión Centro Democrático (UCD) con el 34 \% de los votos, seguida por el Partido Socialista Obrero Español (PSOE), con el $28 \%$ de los votos.

Uno de los temas que más se debatieron en los mensajes de los editoriales y artículos de opinión del $A B C$ y El País, fue la necesidad de llegar a un acuerdo político mediante la firma de un pacto entre los partidos, que garantizara la transición a la democracia en armonía, estabilidad social, política y económica. En octubre de 1977, el presidente de Gobierno, Adolfo Suárez, convocó a los principales líderes políticos de la oposición y a los sindicatos obreros para superar la crisis. Se firmaron los Pactos de Moncloa.

Otro de los tópicos que también se trataron con gran vehemencia en los mensajes periodísticos de los editoriales y artículos de opinión del diario $A B C$ y del El País, fue el tema de promulgar una Constitución que se acomodara a los nuevos tiempos. Como consecuencia se elaboró una nueva Constitución, que fue aprobada en diciembre de 1978.

\section{Consideraciones finales}

En términos generales podría decirse, con todas las interrogantes que se formularon a lo largo de este trabajo de investigación, que la Ley de Prensa de 1966 dio las pautas iniciales para abrir los espacios a la libertad de expresión, para que se rompieran los viejos moldes de una prensa oficialista y complaciente con los in- 
tereses de la clase en el poder, y en este sentido, el mensaje periodístico, liberado de algunas ataduras, influyó decisivamente en el proceso de transición a la democracia.

En esta Tesis Doctoral no se ha pretendido agotar el espacio sobre el papel de la prensa en las etapas de la transición a la democracia del caso español, definitivamente es un campo abierto para seguir investigando y aprovechar la experiencia que en este sentido se ha logrado, sobre todo en los países latinoamericanos, como México o Chile, por citar algunos, que se encuentran en una etapa de transición a la democracia, donde la prensa definitivamente tiene que romper con los viejos esquemas de un periodismo oficialista y abrir los espacios para un verdadero cambio.

\section{Referencias bibliográficas}

Alférez, A. (1986): El cuarto poder en España, Barcelona: Plaza \& Janés.

Aron, R. (1981): Max Weber (El político y el científico), Madrid: Alianza Editorial.

Barbosa-Ramírez, A.R. (1975): La estructura económica de la nueva España. (1519/1810), México: Siglo XXI.

Barrera, C. (1995): Periodismo y franquismo (de la censura a la apertura), Barcelona: Ediciones Internacionales Universitaria.

Beatty, J. (1998): El mundo según Peter Drucker, México: Hermes.

Bardin, L. (1996): Análisis de contenido, Madrid: Ediciones Akal.

Beck Ulrich (1999): Un nuevo mundo feliz (La precariedad del trabajo en la era de la globalización), Barcelona: Paidós.

Fraga Iribarne, M. (1980): Memoria breve de una vida pública, Barcelona: Planeta.

Fukuyama, F. (1994): El fin de la historia y el último hombre, Barcelona: Planeta.

- (1994): La gran ruptura, Barcelona: Ediciones B.

Galbraith, J. K. (1998): Historia de la economía, México: Planeta Mexicana.

García Lahiguera, F. (1975): Investigación de mercados, Bilbao: Deusto.

Gómez Aparicio, P. (1981): Historia del periodismo español, Madrid: Editorial Nacional de Madrid.

Guéhenno, J.M. (1999): El porvenir de la libertad, (La democracia en la época de la globalización), Barcelona: Paidós.

Innis, H. (1950): Empire and Comunication, Londres: Clarendon.

Hobbes, T. (1995): Leviatán, Madrid: Alianza Universitaria.

Jonson, L.M. (1975): El nuevo periodismo, Buenos Aires: Troquel.

Imbert, G. y Vidal Beneyto, J. (coords.), (1986): El País o la referencia dominante, Barcelona: Mitre.

$202 \mid n^{\circ} 8$ | doxa.comunicación 
Luca De Tena, T. (1993): Franco, si pero... Confesiones profanas, Barcelona: Planeta.

López Hidalgo, A. (1996): Las columnas del Periódico, Madrid: Ediciones Libertarias/Prodhufi.

McLuhan, M. (1998): La Galaxia Gutenberg (Génesis del homo Typographicus), Barcelona: Círculo de Lectores.

Matterland, A. (1997): ¿Cómo resistir la colonización de las mentes?, Madrid: Pensamiento Crítico, Temas Debate.

Martínez Alberto, J.L. (1974): El ocaso del periodismo, Barcelona: Libros de Comunicación Global.

Morin, V. (1987): Tratamiento periodístico de la información, Barcelona: Mouton y Co. A.T.E.

Morley Sylvanus, G. (1987): La civilización maya, México: Fondo de Cultura Económica.

Núñez Ladevéze, L. (1995): Ideología y libertad, Madrid: Noesis.

Perelman, CH., y Olbrechts-Yyteca, L. (1989): Tratado de la argumentación (La nueva retórica), Madrid: Gredos.

Popper, K. (1998): La sociedad abierta y sus enemigos, Barcelona: Paidós.

Prados y López, M. (1943): Ética y estética del periodismo español, Madrid: Espasa-Calpe.

Ruiz Collantes, F.X. (2000): Retórica creativa (Programas de la idealización Publicitaria), Barcelona: Universidad Autónoma de Barcelona.

Sampson, A. (1983): Los bancos y la crisis mundial, México: Grijalvo.

Santamaría Suárez, L. (1997): Géneros para la persuasión en periodismo, Madrid: Fragua.

Sartori, G. (1994): La democracia después del comunismo, Madrid: Alianza Editorial.

- (1998): Homo Videns (La sociedad teledirigida), Madrid: Taurus.

Soros, G. (1999): La crisis del capitalismo global (La sociedad abierta en peligro), Madrid: Debate.

Tacliacarme, A. (1960): La democracia en América, México: Fondo de Cultura Económica.

Toffler, A. (1987): El shock del futuro, México: Editorial Diana.

Vázquez y López Riva, (1962): La investigación social, Madrid: OPE.

Villoria Mendieta, M. (1997): La modernización de la administración como instrumento al servicio de la democracia, Madrid: BOE.

Villar, P. (1999): Historia de España, Madrid: Editorial Crítica.

- (2000): La guerra civil española, Barcelona: Crítica.

Weston, A. (1996): Las claves de la argumentación, Barcelona: Ariel. 